\title{
De la bande dessinée comme de la littérature Sylvain Rhéault
}

\section{Introduction}

Depuis quelques années, on trouve de nouveaux types de bandes dessinées sur les étagères des librairies. Ce ne sont pas des albums cartonnés, il n'y a pas de couleur, le nombre de page est assez élevé et le format est plus petit. Mon hypothèse, c'est que les nouvelles productions des éditions alternatives de la bande dessinée démontrent des caractéristiques semblables à celles de la littérature.

Pour explorer la question, je vais examiner l'œuvre Persepolis de Marjane Satrapi comme œuvre représentative d'un changement de cap qui rapproche la bande dessinée et de la littérature. Cette bande dessinée autobiographique, qui se démarque nettement de la production traditionnelle, a été publiée par les éditions de l'Association, dont les choix éditoriaux sont pour le moins hardis. En effet, comme l'explique Bart Beaty dans son livre Unpopular Culture, le projet de cette maison d'édition alternative fondée en 1990 par un groupe de jeunes bédéistes consiste à viser l'art plutôt que les ventes et la pérennité plutôt que la popularité. On compte parmi les fondateurs Jean-Christophe Menu, Lewis Trondheim et quelques autres.

En gros, Persepolis, c'est l'autobiographie de Marjane Satrapi, depuis ses premières années à l'école jusqu'à son départ pour la France, vers 22 ans. Marjane a passé son adolescence en Autriche, où elle s'est retrouvée dans la rue. De retour en Iran, elle fait face à l'intégrisme, elle étudie les Beaux-Arts, se marie puis divorce. On dit que l'originalité de l'œuvre vient de l'interprétation naïve des événements par une petite fille. Afin de comprendre le monde, la petite Marjane s'invente des personnages, puis elle apprend progressivement à décoder les masques des adultes. C'est un apprentissage qui dure toute sa vie, et ses parents sont des éducateurs hors pair en ce sens. Marjane développera un regard d'adolescente rebelle et un engagement de femme qui cherche à changer la société.

Je propose d'analyser les caractéristiques de Persepolis en tant qu'œuvre représentative de cette nouvelle école de bande dessinée. Seront examinés entre autres le statut de l'auteur, la diversité des genres, ainsi que les choix de formes visuelles et de format qui peuvent être mis en parallèle avec ceux de la littérature contemporaine. 


\section{Statut d'artiste pour le bédéiste}

Michael Farr rapporte dans The Adventures of Hergé Creator of Tintin que Hergé, dans les années trente, est rapidement devenu une célébrité médiatique. La gloire et la richesse acquises par l'auteur belge ont certainement inspiré les jeunes de Belgique et du reste de la francophonie à envisager sérieusement la carrière de bédéiste. En particulier, les étudiants des écoles de beaux-arts ont été réceptifs à cette nouvelle vocation. Avec leur connaissance académique du dessin, ces étudiants ont produit des personnages bien proportionnés évoluant au sein de décors splendides dans des pages bien équilibrées et remplies de détails. En France et en Belgique, il était dorénavant possible pour un auteur de BD talentueux et productif de faire fortune, à condition que sa série devienne populaire. Plus un auteur avait de succès commercial, plus il était adulé. On peut penser à Peyo, le créateur des Schtroumpfs, ou à Uderzo, le dessinateur d'Astérix, qui, en plus de toucher les droits sur les albums vendus, ont pu engranger davantage d'argent lorsque leurs séries ont été adaptées à la télévision ou au cinéma.

C'est en partie en réaction à cet état de choses que L'Association a été fondée. Les jeunes éditeurs s'étaient donné pour mission de publier des auteurs dont les productions manifesteraient avant tout un souci d'innovation esthétique. De nouveaux artistes comme David B. ou Guy Delisle, avec leurs styles innovateurs et uniques, ont ainsi pu voir leurs œuvres publiées.

Dorénavant, tout comme pour l'auteur de littérature, le bédéiste est invité à choisir une voie. Il peut s'engager sur le chemin du succès commercial en imitant les recettes éprouvées de ses prédécesseurs, ou s'aventurer dans l'expérimentation en risquant l'oubli et la pauvreté. Cette dernière voie constitue le « clan » des «vrais » artistes. Ce sont ceux qui, de leur vivant, vendent généralement peu mais sont reconnus par la suite pour leur contribution à l'art. On peut penser à un artiste maudit comme Rimbaud. Par ailleurs, Sartre, au-delà de la recherche esthétique, réclamait des auteurs une intentionnalité qu'il appelait un «engagement » dans Qu'est-ce que la littérature. Une œuvre comme Persepolis, avec ses prises de conscience politiques, s'accorde parfaitement bien avec la vision sartrienne de la littérature. Si Persepolis n'atteint pas les tirages des albums de BD les plus populaires, il est indéniable que l'auteur, Marjane Satrapi, est aujourd'hui considérée comme une artiste originale ayant des choses importantes à raconter.

\section{Diversification des genres pour le médium de la bande dessinée}

Pour Scott McCloud, la bande dessinée est d'abord un médium, au même titre que le 
cinéma, le théâtre, le roman et la télévision. En principe, chacun de ces média peut accommoder différents genres et différents registres, comme l'aventure, la comédie romantique, la biographie, la science-fiction, le roman policier, etc. Pourtant, malgré la capacité d'accommodement des média pour les genres, aux États-Unis, dans les années cinquante, les récits de super héros avaient si bien envahi le champ des bandes dessinées, et pendant si longtemps, que le public américain en était venu à confondre aisément le médium avec le genre. À un moindre degré, en Europe, la bande dessinée se voyait malgré tout cantonnée à certains genres qui se vendaient mieux, comme l'aventure (Tintin), l'humour (Astérix) et l'érotisme (Barbarella). Par contre, personne ne décrirait le roman comme le véhicule d'un seul genre parce que tous les romans ne sont pas des polars ou des idylles à l'eau de rose. On dira la même chose du cinéma ; ce n'est pas que des films d'action. Dans les romans et au grand écran, le public peut apprécier une grande variété de genres.

Si la richesse d'un médium repose sur la diversité des genres qui s'y étalent, il faut reconnaître que le neuvième art a fait des progrès considérables depuis une vingtaine d'années. Notons en particulier l'apparition de l'autobiographie en bande dessinée, avec des auteurs comme David B. (Le Haut Mal) ou Guy Delisle (Pyongyang). Le genre n'avait jamais été exploré de manière aussi sincère autrefois. Si Gotlib affichait volontiers son image dans Rubrique à Brac, en revanche il ne dévoilait rien de son intimité. L'autobiographie, comme le remarque Bart Beaty, représente une source d'authenticité pour le lecteur, qui établit ainsi avec l'auteur un rapport plus intime. C'est justement à ce genre de prospection que se livre Marjane Satrapi.

D'autre part, s'inspirant du modèle de l'Oulipo, L'Association a lancé l'aventure de l'Oubapo. Il s'agit de créer des planches à partir de certaines contraintes pré-établies. Par exemple, mettre des cases sur des dés et improviser une histoire en les roulant, reprendre une planche en insérant des cases entre les cases, appliquer à une planche le dialogue d'une autre planche, etc. Ce genre d'expérimentation contribue à donner au médium de la bande dessinée une diversité de genres comparable à celle du roman.

\section{Métamorphoses du format $\mathbf{4 8 C C}$}

Tout comme la BD, le roman prend le plus souvent la forme du livre. On ne discutera pas ici des possibilités offertes par les média électroniques. Hergé, dès ses tout débuts, a eu le bonheur d'être publié par les éditions Casterman qui avaient opté de relier les albums de Tintin 
avec une couverture cartonnée, à l'instar des beaux livres pour enfants de ses autres collections. La couverture cartonnée suggérait un item durable que l'on peut collectionner. Le format a plu au public et s'est imposé aux productions à venir. Quant au nombre de pages des albums francobelges, cela varie entre 44 et 62 pages. Jean-Christophe Menu, dans son essai Plates-bandes, y réfère de manière un peu condescendante comme le $48 \mathrm{CC}$, soit un album de $\mathbf{4 8}$ pages en Couleur et avec couverture Cartonnée.

Mais faisons un bref saut dans le passé. Dès les débuts de l'industrie de la bande dessinée franco-belge, Hergé, encore lui, avait établi des standards assez élevés. Soucieux d'offrir des planches de la plus grande qualité possible, le père de Tintin recourait à une abondance de documents photographiques pour les décors et faisait prendre des poses à ses collaborateurs afin de donner aux corps des personnages des postures physiquement correctes. Tout cela a contribué à développer un style narratif très efficace connu sous le nom de «ligne claire », résultat de la recherche constante d'une lisibilité maximale. Hergé a ajouté très tôt de la couleur à ses dessins, toujours dans le souci d'en accroître la lisibilité. Tout comme pour le reste, la mise en couleur des planches a été faite avec soin.

Afin de signaler clairement sa rupture avec les productions des héritiers d'Hergé, dont les planches sont caractérisées par la précision technique du dessin ainsi que la mise en couleur, L'Association s'est évertuée à publier des œuvres où le noir et blanc est systématique et où l'expressivité du trait l'emporte sur l'exactitude académique. Persepolis, par exemple, propose une vision unique, dans un style dépouillé qui n'est pas sans rappeler les illustrations persanes. Le dessin y dégage une subjectivité et une intimité qui servent admirablement le propos autobiographique. Ce dessin, qui va à l'essentiel, donc moins détaillé mais plus nerveux, semble chercher à se distinguer non seulement d'une certaine tradition de la bande dessinée, mais aussi de l'art visuel ou de l'illustration pour ainsi, à défaut d'autres directions, se rapprocher d'un médium narratif comme le roman. Au risque d'avancer une grossière évidence, il faut mentionner que la littérature s'est toujours contentée du noir et blanc.

Des tentatives de publier des albums avec couverture souple avaient été tentées par le passé. On peut penser entre autres à ceux produits par les éditions Dupuis dans les années 1970. Si les consommateurs néerlandais en raffolaient, en revanche les collectionneurs franco-belges leur préféraient la couverture cartonnée, même si la dimension des planches restait la même.

De même, la revue (À suivre), qui a paru de 1978 à 1997, avait aussi expérimenté avec le 
noir et blanc. Les planches pré-publiées étaient ensuite réunies en album avec couverture souple de plus de 100 pages. Les aventures de Corto Maltese ont trouvé là un champ fertile pour se développer.

Outre le style du trait, la couleur des planches et le nombre de pages, la dimension des albums est aussi un aspect qui a fait l'objet d'expérimentation. Ici, la mise en parallèle avec l'histoire du livre peut être instructive. À partir de 1930, les éditeurs offraient à la littérature un nouveau véhicule avec le livre de poche (Wikipédia). Plus petit que le livre traditionnel, avec une couverture souple, le format de poche était produit pour la consommation plutôt que pour la collection. Le format a si bien marché que la majorité des romans que l'on achète aujourd'hui l'ont adopté. De même, pour les albums de bandes dessinées, le format aussi a fait l'objet d'expérimentations commerciales. Dargaud avait lancé sur le marché au début des années 1980 un format plus petit, baptisé 16/22 en référence à la hauteur et à la largeur du volume en centimètres, qui n'a pas connu beaucoup de succès. Les éditeurs ont aussi tenté de convertir la bande dessinée au format livre de poche, mais les puristes s'étaient levés contre les découpages abominables qu'il fallait faire subir aux planches de BD afin de les faire tenir sur des pages trop petites.

Un facteur important dans la métamorphose du format, dont il faut absolument tenir compte, c'est l'invasion des mangas, qui a vraiment commencé en France vers 1990, avec les Éditions Glénat. Les mangas sont aujourd'hui immensément populaires non seulement en France mais aussi dans le reste du monde. Si les mangas sont pré-publiés dans des revues de grand format, leur réimpression se fait en format tankobon, semblable en dimension à un livre de poche. Mais la planche de manga s'y prête bien, puisqu'il n'y a pas plus d'une, deux ou trois bandes au maximum. Condenser les planches franco-belges traditionnelles, avec quatre bandes par planche, se révèle une opération plus complexe. Notons que les mangas les plus populaires sont réimprimés en format aizoban ou waidoban, similaire en dimension au graphic novel américain, mais plus épais.

Les versions les plus récentes de l'édition complète de Persepolis ont une couverture souple, se présentent en noir et blanc, comptent plus de 200 pages et adoptent un format semblable au graphic novel américain. On ne pourrait se distancer davantage du format 48CC. 


\section{Conclusion}

Le bédéiste peut choisir une voie tout comme un auteur littéraire et employer le genre qui convient le mieux à son propos. C'est un progrès notable. D'autre part, les métamorphoses du format traditionnel de l'album de bande dessinée dans un format nouveau qui peut être considéré comme l'équivalent du livre de poche, entraînent certaines conséquences. Comme la bande dessinée fait l'objet d'une publication directe, sans prépublication, les auteurs doivent dorénavant compter avec les contraintes et avantages du nouveau format. Si l'album 48CC, plus grand, invitait à regarder des images en couleur, en revanche le nouveau format, plus petit, invite à lire une histoire. De même, le nouveau format, avec un plus grand nombre de pages (200+ au lieu de 44), porte à une lecture plus soutenue, à des développements plus longs. Si la couverture cartonnée poussait à la collection, la couverture souple exhorte à donner de l'importance au contenu plutôt qu'à l'objet. Enfin, même si c'est un peu hors de notre propos, il faut mentionner que le nouveau format côtoie harmonieusement les livres classiques.

Le médium de la bande dessinée a fait son entrée dans le monde franco-belge comme un livre d'images de luxe pour enfants. Nous en sommes aujourd'hui à un point où les nouvelles œuvres produites apparaissent telles qu'elles l'auraient été si la bande dessinée avait fait son entrée par la porte du livre de littérature plutôt que par les publications pour la jeunesse.

\section{Ouvrages cités}

Beaty, Bart. UNpopular Culture - Transforming the European Comic Book in the 1990s. Toronto : U of Toronto P, 2007.

Farr, Michael. The Adventures of Hergé Creator of Tintin. Londres : Murray, 2007.

McCloud, Scott. Understanding Comics. New York : Harper Collins, 1993.

Menu, Jean-Christophe. Plates-bandes. Paris : L'Association, 2005.

Sartre, Jean-Paul. Qu'est-ce que la littérature? Paris: Gallimard, 1948.

Satrapi, Marjane. Persepolis. Paris : L'Association, 2007 [2000-2003].

«Livre de Poche ». Wikipedia. 2010. 25 octobre 2010.

<http://fr.wikipedia.org/wiki/Livre_de_poche/>. 